\title{
On a Balanced Property of Derangements
}

\author{
Miklós Bóna * \\ Submitted: Jun 6, 2006; Accepted: Oct 30, 2006; Published: Nov 6, 2006 \\ Mathematics Subject Classification: 05A16
}

\begin{abstract}
We prove an interesting fact describing the location of the roots of the generating polynomials of the numbers of derangements of length $n$, counted by their number of cycles. We then use this result to prove that if $k$ is the number of cycles of a randomly selected derangement of length $n$, then the probability that $k$ is congruent to a given $r$ modulo a given $q$ converges to $1 / q$. Finally, we generalize our results to $a$-derangements, which are permutations in which each cycle is longer than $a$.
\end{abstract}

\section{Introduction}

Let $c(n, k)$ denote the number of permutations of length $n$ with $k$ cycles. The numbers $c(n, k)$ are then called the signless Stirling numbers of the first kind. It is well-known [3] that

$$
C_{n}(x)=\sum_{k=1}^{n} c(n, k) x^{k}=x(x+1) \cdots(x+n-1) .
$$

Setting $x=-1$, this shows that there are as many permutations of length $n$ with an odd number of cycles as there are with an even number of cycles.

If, instead of considering the sum of all Stirling numbers $c(n, k)$ so that $n$ is fixed and $k$ belongs to a certain residue class modulo 2, we consider the sum of the Stirling numbers $c(n, k)$ so that $n$ is fixed and $k$ belongs to a certain residue class modulo $q$, the result is a little bit less compact. These sums will no longer be equal to $n ! / q$, but it will be true that as $n$ goes to infinity, the limit of any of these $q$ sums divided by $n$ ! will converge to $1 / q$. We will prove this fact in this paper, as a way to illustrate our techniques.

A derangement is a permutation with no fixed points (cycles of length 1 ). It is wellknown [2] that number of derangements of length $n$ is $D(n)=n ! \sum_{i=0}^{n} \frac{(-1)^{i}}{i !}$, which is the

${ }^{*}$ University of Florida, Gainesville FL 32611-8105. Partially supported by an NSA Young Investigator Award. Email: bona@math.ufl.edu.

THE ELECTRONiC JOURNAl OF COMBINATORICS 13 (2006), \#R102 
integer closest to $n ! / e$. Let $d(n, k)$ be the number of derangements of length $n$ with $k$ cycles. The numbers $d(n, k)$ are not nearly as well-studied as the numbers $c(n, k)$, but the following important fact is known about them. The result is due to E. R. Canfield [6], and a more general version can be found in [5].

Theorem 1.1 Let $n$ be a positive integer. Let

$$
D_{n}(x)=\sum_{k=1}^{\lfloor n / 2\rfloor} d(n, k) x^{k}
$$

be the ordinary generating function of the numbers of derangements of length $n$ according to their number of cycles.

Then all roots of the polynomial $D_{n}(x)$ are real and non-positive.

In this paper, we will prove that derangements also have the "balanced" property that we described for permutations. That is, if $q$ and $r$ are fixed integers so that $0 \leq r<q$, and $k$ is the number of cycles of a randomly selected derangement of length $n$, then the probability that $k$ is congruent to $r$ modulo $q$ will converge to $1 / q$ when $n$ goes to infinity.

The proof will proceed as follows. We will show that for any negative integer $-t$, there exists a positive integer $N$ so that if $n>N$, then $D_{n}(x)$ has a root that is very close to -1 , a root that is very close to -2 , and so on, up to a root that is very close to $-t$. So these $t+1$ roots of $D_{n}(x)$ (including the obvious root at 0 ) will be very close to the first $t+1$ roots of $C_{n}(x)$, that is, to the integers $0,-1, \cdots,-t$.

Finally, we will generalize both results to $a$-derangements, which are permutations in which each cycle is longer than $a$.

\section{The roots of the generating function of derange- ments}

The goal of this section is proving the announced result on the roots of $D_{n}(x)$. We will need the following lemma.

Lemma 2.1 For all negative integers $-t$, there exists a polynomial $f_{t}$ of degree $t$ so that for all positive integers $n$,

$$
D_{n}(-t)=f_{t}(n) \cdot t^{n}
$$

Proof: By the Principle of Inclusion-Exclusion, the numbers $d(n, k)$ can be expressed by the signless Stirling numbers of the first kind $c(n, k)$ in the following way

$$
d(n, k)=\sum_{i=0}^{k}\left(\begin{array}{l}
n \\
i
\end{array}\right) c(n-i, k-i)(-1)^{i} .
$$


Indeed, a derangement is a permutation that has no 1-cycles, and the right-hand side counts permutations of length $n$ with $k$ cycles according to the number of their 1-cycles.

Multiply both sides by $(-t)^{k}$ and sum over all positive integers $k \leq n$, to get

$$
D_{n}(-t)=\sum_{k=1}^{n}\left(\sum_{i=0}^{k}\left(\begin{array}{l}
n \\
i
\end{array}\right) c(n-i, k-i)(-1)^{i}\right)(-t)^{k} .
$$

After changing the order of summation, this yields

$$
\begin{aligned}
D_{n}(-t) & =\sum_{i=0}^{n}(-1)^{i}(-t)^{i}\left(\begin{array}{c}
n \\
i
\end{array}\right)\left(\sum_{k=i}^{n} c(n-i, k-i)(-t)^{k-i}\right) \\
& =\sum_{i=0}^{n} t^{i}\left(\begin{array}{c}
n \\
i
\end{array}\right)\left(\sum_{k=i}^{n} c(n-i, k-i)(-t)^{k-i}\right) .
\end{aligned}
$$

The crucial observation is that the inner sum of the right-hand side is 0 as long as $n-i \geq$ $t+1$. Indeed, note that the inner sum is nothing else but the left-hand side of (1) with $n-i$ and $k-i$ playing the roles of $n$ and $k$. Then (1) tells us that this expression vanishes for all negative integers $-t$ not smaller than $-(n-i)+1$.

Therefore, (4) simplifies to

$$
D_{n}(-t)=\sum_{i=n-t}^{n} t^{i}\left(\begin{array}{l}
n \\
i
\end{array}\right)\left(\sum_{k=i}^{n} c(n-i, k-i)(-t)^{k-i}\right),
$$

the importance of which is that now we are dealing with summations with a bounded number of terms. Indeed, the outer sum has $t+1$ terms, and for each value of $i$, the inner sum has at most $t+1$ terms. Finally, as $\left(\begin{array}{c}n \\ i\end{array}\right)=\left(\begin{array}{c}n \\ n-i\end{array}\right)$ and $n-i \leq t$, this binomial coefficient is a polynomial function of degree at most $t$. To summarize, the right-hand side is a sum of at most $(t+1)^{2}$ terms, each of which is a polynomial function of $n$ multiplied by a function of $t$ of the form $t^{m}$, with $n-t \leq m \leq n$. As $n-i \leq t$, the Stirling numbers $c(n-i, k-i)$ are bounded and can be treated as constants. This proves our statement. $\diamond$

Example 2.2 If $-t=-1$, then we get the well-known result that $D_{n}(-1)=1-n$. In other words, the number of derangements of length $n$ that are odd permutations and the number of derangements of length $n$ that are even permutations differs by $(n-1) . R$. Chapman [7] gave a bijective proof for this result.

Now we are in a position to prove our result on the roots of $D_{n}(x)$.

Theorem 2.3 For every negative integer $-t$, and every $\epsilon>0$, there exists a positive integer $N$ so that if $n>N$, then $D_{n}(x)$ has a root $x_{t}$ satisfying $\left|-t-x_{t}\right|<\epsilon$. 
That is, for large $n$, there will be a root of $D_{n}(x)$ that is almost -1 , a root that is almost -2 , and so on, up to a root that is almost $-t$.

Proof: (of Theorem 2.3) Let $a_{n}=d(n,\lfloor n / 2\rfloor)$ be the leading coefficient of $D_{n}(x)$. Then

$$
\frac{D_{n}(x)}{a_{n}}=\prod_{i=1}^{\lfloor n / 2\rfloor}\left(x-x_{i}\right)
$$

where the $x_{i}$ are the roots of $D_{n}(x)$. Then it follows from the Pigeon-hole Principle that

$$
\sqrt[\lfloor n / 2\rfloor]{\left|\frac{D_{n}(x)}{a_{n}}\right|} \geq \min _{i}\left|x-x_{i}\right|
$$

for all real numbers $x$. In particular, for $x=-t$, Lemma 2.1 yields

$$
\sqrt[\lfloor n / 2\rfloor]{\frac{\left|f_{t}(n) t^{n}\right|}{a_{n}}} \geq \min _{i}\left|-t-x_{i}\right|
$$

for some polynomial $f_{t}$ of degree $t$. (Note that no absolute value sign is needed for the denominator of the left-hand side as a $a_{n}$ is always a positive integer.)

Now if $n$ is even, then a derangement with $n / 2$ cycles consists of 2 -cycles only, and the number of such derangements is $a_{n}=(n-1)(n-3) \cdots 1$. If $n$ is odd, then a derangement with $(n-1) / 2$ cycles consist of one 3 -cycle and $(n-3) / 2$ cycles of length two. The number of such derangements is $a_{n}=\frac{n-1}{3} n(n-2) \cdots 1$. Then the well-known Stirling's formula stating that $n ! \sim\left(\frac{n}{e}\right)^{n} \sqrt{2 \pi n}$ shows that in both cases, $a_{n}$ grows much faster than $D_{n}(-t)$. Indeed if $2 m-1$ is the largest odd integer so that $2 m-1 \leq n$, then by Stirling's formula

$$
(2 m-1) ! !=1 \cdot 3 \cdots(2 m-1) \sim \sqrt{2} \cdot\left(\frac{4 m}{e}\right)^{m} .
$$

Therefore, the left-hand side of (9) converges to 0 as $n$ goes to infinity, and consequently, so does the right-hand side. This proves our claim. $\diamond$

\section{Balanced Properties}

In this section, we prove the announced balanced properties for permutations, derangements, and $a$-derangements. We start with the easier problem of permutations.

\subsection{Permutations}

Theorem 3.1 Let $q$ be a positive integer, and let $r$ be a non-negative integer so that $0 \leq r<q$. Then

$$
\lim _{n \rightarrow \infty} \frac{\sum_{j=0}^{(n-r) / q} c(n, r+j q)}{n !}=\lim _{n \rightarrow \infty} \frac{c(n, r)+c(n, q+r)+\cdots+c(n, q\lfloor(n-r) / q\rfloor+r)}{n !}=\frac{1}{q} .
$$


In other words, for large $n$, the number of cycles of a randomly selected $n$-permutation is roughly equally likely to be congruent to each $r$ modulo $q$.

Proof: In order to simplify the presentation, let us assume first that $n$ is divisible by $q$, and that $r=0$. Then the statement to be proved reduces to

$$
\lim _{n \rightarrow \infty} \frac{\sum_{j=1}^{n / q} c(n, j q)}{n !}=\lim _{n \rightarrow \infty} \frac{c(n, q)+\cdots+c(n, n)}{n !}=\frac{1}{q} .
$$

Let $w$ be a primitive $q$ th root of unity, and consider $C_{n}(w)$. Then $w^{k}=1$ if and only if $k$ is a multiple of $q$; otherwise $w^{k}$ is not a positive real number. Now consider the sum

$$
\begin{aligned}
S(w) & =C_{n}(1)+C_{n}(w)+C_{n}\left(w^{2}\right)+\cdots+C_{n}\left(w^{q-1}\right) \\
& =\sum_{t=0}^{q-1} C_{n}\left(w^{t}\right) \\
& =\sum_{t=0}^{q-1}\left(\sum_{k=1}^{n} c(n, k)\left(w^{t}\right)^{k}\right) \\
& =\sum_{k=1}^{n} c(n, k)\left(\sum_{t=0}^{q-1}\left(w^{k}\right)^{t}\right)
\end{aligned}
$$

Using the summation formula of a geometric progression, we get that

$$
\sum_{t=0}^{q-1}\left(w^{k}\right)^{t}=\left\{\begin{array}{l}
0 \text { if } w^{k} \neq 1, \text { that is } q \nmid k, \\
q \text { if } w^{k}=1, \text { that is, } q \mid k .
\end{array}\right.
$$

Therefore, (11) reduces to

$$
\begin{aligned}
& S(w)=q \cdot \sum_{j=1}^{n / q} c(n, j q), \\
& \frac{S(w)}{q \cdot n !}=\frac{\sum_{j=1}^{n / q} c(n, j q)}{n !} .
\end{aligned}
$$

So in order to find the approximate value of $\left(\sum_{j=1}^{n / q} c(n, j q)\right) / n$ !, it suffices to find the approximate value of $S(w) / n$ !. We will find the latter by showing that for large $n$, the contribution of $C_{n}\left(w^{t}\right) / n$ ! to $S(w) / n$ ! is negligible unless $w^{t}=1$. This is the content of the next lemma. Once the lemma is proved, Theorem 3.1 will follow since $C_{n}(1)=n$ !.

Lemma 3.2 Let $v \neq 1$ be a complex number satisfying $|v|=1$. Then

$$
\lim _{n \rightarrow \infty} \frac{C_{n}(v)}{n !}=0
$$


Proof: (of Lemma 3.2) Let $v=a+b i$, and let $g_{n}(v)=\frac{C_{n}(v)}{n !}$. It suffices to show that $\lim _{n \rightarrow \infty}\left|g_{n}(v)\right|^{2}=0$. Recalling (1), we obtain

$$
\begin{aligned}
\left|g_{n}(v)\right|^{2} & =\prod_{j=1}^{n-1} \frac{a^{2}+2 a j+j^{2}+b^{2}}{(j+1)^{2}}=\prod_{j=1}^{n-1} \frac{1+2 a j+j^{2}}{(j+1)^{2}} \\
& =\prod_{j=1}^{n-1} \frac{2 a j+j^{2}}{(j+1)^{2}} \cdot \prod_{j=1}^{n-1} \frac{1+2 a j+j^{2}}{2 a j+j^{2}} \\
& =\prod_{j=1}^{n-1} \frac{1+2 a j+j^{2}}{2 a j+j^{2}} \cdot \prod_{j=1}^{n-1} \frac{j+2 a}{j+1} \cdot \prod_{j=1}^{n-1} \frac{j}{j+1} \\
& =\frac{1}{n} \cdot \prod_{j=1}^{n-1} \frac{1+2 a j+j^{2}}{2 a j+j^{2}} \cdot \prod_{j=1}^{n-1} \frac{j+2 a}{j+1}
\end{aligned}
$$

The first infinite product in (18) is convergent since

$$
\log \left(\frac{1+2 a j+j^{2}}{2 a j+j^{2}}\right)=\log \left(1+\frac{1}{2 a j+j^{2}}\right)<\frac{1}{j^{2}},
$$

and $\sum_{j \geq 1} \frac{1}{j^{2}}$ is convergent. If $a>0.5$, then the second infinite product in (18) in itself is divergent. However, multiplied by the factor $1 / n$ in front it will converge to 0 . Indeed,

$$
\begin{aligned}
\log \left(\prod_{j=1}^{n-1} \frac{j+2 a}{j+1}\right) & =\sum_{j=1}^{n-1} \log \left(\frac{j+2 a}{j+1}\right) \\
& =\sum_{j=1}^{n-1} \log \left(1+\frac{2 a-1}{j+1}\right)<\sum_{j=1}^{n-1} \frac{2 a-1}{j+1} \\
& <(2 a-1) \log n .
\end{aligned}
$$

As $2 a-1<1$, this shows that $\frac{1}{n} \prod_{j=1}^{n-1} \frac{j+2 a}{j+1}$ converges to 0 , proving the claim of Lemma 3.2. $\diamond$

So $C_{n}\left(w^{t}\right) / n$ ! converges to 0 unless $w^{t}=1$, which proves $(10)$.

Finally, let us return to the general case of Theorem 3.1. First, note that if $n$ is divisible by $q$, but $r \neq 0$, then we can use an analogous argument, simply replacing $S(w)$ by

$$
T_{r}(w)=C_{n}(1)+C_{n}(w) w^{-r}+C_{n}\left(w^{2}\right) w^{-2 r}+\cdots+C_{n}\left(w^{q-1}\right) w^{-1(q-1) r} .
$$

Then a computation analogous to (11) yields

$$
T_{r}(w)=\sum_{k=1}^{n} c(n, k)\left(\sum_{t=0}^{q-1}\left(w^{k-r}\right)^{t}\right)
$$


showing that the coefficient of $w^{k}$ in $T_{r}(w)$ is 0 unless $k-r$ is divisible by $q$, in which case this coefficient is $q$. The proof of Theorem 3.1 then follows from Lemma 3.2 as in the $r=0$ case. Finally, if $n$ is not divisible by $q$, then nothing significant changes, (19) and (20) will still hold, completing the proof. $\diamond$

\subsection{Derangements}

Given the form of the generating function $D_{n}(x)$, we can apply the method of the above proof to obtain a similar result for derangements.

Theorem 3.3 Let $r$ be an integer so that $0 \leq r<q$, and let $m=\lfloor n / 2\rfloor$. Then

$$
\lim _{n \rightarrow \infty} \frac{\sum_{j=0}^{m / q} d(n, r+j q)}{D(n)}=\lim _{n \rightarrow \infty} \frac{d(n, r)+d(n, q+r)+\cdots+d(n, q\lfloor(m-r) / q\rfloor+r)}{D(n)}=\frac{1}{q} .
$$

In other words, if $n$ is large enough, then the number of derangements of length $n$ whose number of cycles is congruent to $r$ modulo $q$ will be very close to $1 / q$ times the total number of derangements of length $n$.

Proof: In order to simplify the discussion, let us again assume that $n$ is divisible by $q$, and that $r=0$. The general case will follow from this special case just as in the proof of Theorem 3.1.

In the special case at hand, our task is to prove that

$$
\lim _{n \rightarrow \infty} \frac{\sum_{j=1}^{n / 2 q} d(n, j q)}{D(n)}=\lim _{n \rightarrow \infty} \frac{d(n, q)+\cdots+d(n, n / 2)}{D(n)}=\frac{1}{q} .
$$

Similarly to the case of permutations, let $w$ be a $k$ th primitive root of unity, and set

$$
\begin{aligned}
Z(w) & =D_{n}(1)+D_{n}(w)+D_{n}\left(w^{2}\right)+\cdots+D_{n}\left(w^{q-1}\right) \\
& =\sum_{k=1}^{n / 2} d(n, k)\left(\sum_{t=0}^{q-1}\left(w^{k}\right)^{t}\right) \\
& =q \sum_{j=1}^{n / 2 q} d(n, j q) .
\end{aligned}
$$

We claim that if $w^{t} \neq 1$, then $D_{n}(w) / D(n)$ converges to 0 . This will prove Theorem 3.3 since $D_{n}(1)=D(n)$.

Proposition 3.4 Let $v \neq 1$ be a complex number satisfying $|v|=1$. Then

$$
\lim _{n \rightarrow \infty} \frac{D_{n}(v)}{D(n)}=0
$$


Proof: Let $\epsilon>0$, let $-h$ be a negative integer and let $n$ be so large that $D_{n}(x)$ has a root closer than $\epsilon$ to $-y$, for $-y=-1,-2, \cdots,-h$. Let us call the set of these $h$ roots the good roots. Then, recalling that the roots of $D_{n}(x)$ are all real and non-positive, we get that

$$
\left|\frac{D_{n}(v)}{D(n)}\right|=\left|\frac{D_{n}(v)}{D_{n}(1)}\right|=\frac{\prod_{i=1}^{\lfloor n / 2\rfloor}\left|\left(v-x_{i}\right)\right|}{\prod_{i=1}^{\lfloor n / 2\rfloor}\left|\left(1-x_{i}\right)\right|} \leq \frac{\prod_{i \in L}\left|\left(v-x_{i}\right)\right|}{\prod_{i \in L}\left|\left(1-x_{i}\right)\right|}=: H(v)
$$

where $i \in L$ if $x_{i}$ is a good root. (We are defining $H(v)$ to be the rightmost fraction of the above chain of inequalities.) The last inequality holds since $\frac{\left|v-x_{i}\right|}{\left|1-x_{i}\right|} \leq 1$ holds for each complex number on the unit circle since $x_{i}$ is real and non-positive.

As the good roots $x_{i}$ converge to the negative integers $-1,-2, \cdots,-h$, we see that

$$
H(v) \simeq\left|\frac{C_{h}(v)}{h !}\right|
$$

However, we have proved in Lemma 3.2 that $\left|\frac{C_{h+1}(v)}{(h+1) !}\right|$ converges to 0 when $h$ goes to infinity. It follows from Theorem 2.3 that as $n$ goes to infinity, $h$ will go to infinity. Then (26) and (25) imply our claim. $\diamond$

The proof of Theorem 3.3 is now immediate since all but one term of the right-hand side of (22) has a negligible contribution to $Z(w)$. Therefore, $Z(w) \simeq D_{n}(1)$, and so

$$
\sum_{j=1}^{n / 2 q} d(n, j q) \simeq \frac{Z(w)}{q} \simeq \frac{D_{n}(1)}{q}=\frac{D(n)}{q} .
$$

$\diamond$

\section{$3.3 \quad a$-derangements}

Let us call a permutation $p$ an $a$-derangement if each cycle of $p$ is longer than $a$. So a permutation is a 0-derangement, and a derangement is a 1-derangement. Let $d_{a}(n, k)$ be the number of $a$-derangements of length $n$ with $k$ cycles. Set

$$
D_{n, a}(x)=\sum_{k=1}^{\lfloor n /(a+1)\rfloor} d_{a}(n, k) x^{k}
$$

It is then known [5] that the roots of $D_{n, a}(x)$ are real and non-positive.

Our goal in this subsection is to show that the results that we proved for permutations and derangements hold for $a$-derangements as well. The only part of the argument that needs extra explanation is the analogue of Lemma 2.1 for $a$-derangements. 
Lemma 3.5 For all negative integers $-t$ and for all positive integers $a$, there exists a polynomial $f_{t, a}$ so that for all positive integers $n$,

$$
D_{n, a}(-t)=f_{t, a}(n) \cdot t^{n}
$$

Proof: In order to alleviate notation, for $a \geq 1$, let $m_{a}(i, b)$ denote the number of all permutations of length $b$ that consist of $i$ cycles so that none of these cycles is longer than $a$. For instance, $m_{1}(i, i)=1$, and $m_{2}(i, b)$ is the number of involutions of length $b$ with $i$ cycles. Set $m_{a}(0,0)=1$.

Then the Principle of Inclusion-Exclusion implies that

$$
d_{a}(n, k)=\sum_{i=0}^{k}\left(\sum_{b=i}^{n} c(n-b, k-i) m_{a}(i, b)\left(\begin{array}{l}
n \\
b
\end{array}\right)\right)(-1)^{i} .
$$

Indeed, the right-hand side counts the permutations of length $n$ according to the number of their "bad" (that is, not longer than a) cycles. Those that have $i$ bad cycles are counted according to the total size of those bad cycles.

Multiply both sides by $(-t)^{k}$ and sum over all positive integers $k \leq n$, to get

$$
D_{n, a}(-t)=\sum_{k=1}^{n}\left(\sum_{b=i}^{n} \sum_{i=0}^{k}\left(c(n-b, k-i) m_{a}(i, b)\left(\begin{array}{l}
n \\
b
\end{array}\right)\right)(-1)^{i}\right)(-t)^{k} .
$$

After changing the order of summation, this yields

$$
\sum_{i=0}^{n} t^{i}\left(\sum_{b=i}^{n}\left(\begin{array}{l}
n \\
b
\end{array}\right) m_{a}(i, b)\left(\sum_{k=i}^{n} c(n-b, k-i)(-t)^{k-i}\right)\right) .
$$

Just as in (4), the key observation is again that the innermost sum is 0 as long as $n-i \geq$ $t+1$. Indeed, since $b \geq i$, in that case, the innermost sum is nothing else than the lefthand side of (1). If $b$ is strictly larger than $i$, then there will be a few extra zeros in the sum, as $k-i$ will eventually get bigger than $n-b$.

Therefore, (29) reduces to

$$
D_{n, a}(-t)=\sum_{i=n-t}^{n} t^{i}\left(\sum_{b=i}^{n}\left(\begin{array}{l}
n \\
b
\end{array}\right) m_{a}(i, b)\left(\sum_{k=i}^{n} c(n-b, k-i)(-t)^{k-i}\right)\right) .
$$

Just as we used (6) to prove that $D_{n}(-t)$ was of the form $f_{t}(n)(-t)^{n}$, we can use (30) to prove our lemma. Indeed, as $k$ and $b$ are both at least as large as $i$, and $i \geq n-t$, the right-hand side of $(30)$ is the sum of no more than $(t+1)^{3}$ terms. We claim that each of these terms is of the right form, that is, a power of $t$ times a polynomial function of $n$. Indeed, let us fix $i$ and $b$ within the allowed limits, that is, $n-t \leq i \leq b \leq n$. Then $\left(\begin{array}{l}n \\ b\end{array}\right)=\left(\begin{array}{c}n \\ n-b\end{array}\right)$ is a polynomial function of $n$ that is of degree at most $t$. Finally, $m_{a}(i, b)$ is the number of permutations of length $b$ with $i$ cycles, each of which is at most of length 
$a$. That means that if the permutation $p$ is counted by $m_{a}(i, b)$, then the cycle type of $p$ is very restricted. Indeed, if $p$ has $g_{j}$ cycles of length $j$ for $j \in\{1,2, \cdots, a\}$, then $\sum_{j=1}^{a} j g_{j}=b$ and $\sum_{j=1} g_{j}=i$. As $i \leq b \leq n \leq i+t$, this means that $p$ can have at most $t$ cycles that are not singleton cycles. It is well-known (see for instance [3]) that the number of possibilities for $p$ with the given cycle type is

$$
T_{g_{1}, g_{2}, \cdots, g_{a}}=\frac{b !}{g_{1} ! \cdot g_{2} ! \cdots g_{a} ! \cdot 1^{g_{1}} \cdot 2^{g_{2}} \cdots \cdots a^{g_{a}}} .
$$

However, as we said two sentences earlier, $g_{1} \geq n-t$. Recall from the previous paragraph that $n-t \leq i \leq b \leq n$. This implies that $b$ is equal to one of $n, n-1, \cdots n-t$. Then it follows that for this fixed $i$ and $b$, and each allowed cycle type $\left(g_{1}, g_{2}, \cdots, g_{a}\right)$, the function $T_{g_{1}, g_{2}, \cdots, g_{a}}$ is a polynomial function of $n$. Finally, the number of all allowed cycle types for this fixed $b$ and $i$ is not more than the number of partitions of $b$ into exactly $i$ parts, that is, the number of partitions of $b-i$, which is not more than $p(t)$. Therefore, for any fixed $b$ and $i$, the function $m_{a}(i, b)$ is the sum of a bounded number of polynomial functions of $n$, and as such is a polynomial function of $n$. This proves the lemma. $\diamond$

Note that it would have been somewhat simpler to prove the slightly weaker (but sufficient) result that $\left|D_{n, a}(-t)\right| \leq f_{n, a}(t) t^{n}$. Indeed, we could have just pointed out that $m_{a}(i, b) \leq c(b, i) \leq c(n, i)$, and refer to the well-known fact (see for instance [1], Exercise 4 of Chapter 6 ) that for any fixed $u$, the function $c(n, n-u)$ is a polynomial function of degree $u+1$. However, we preferred a more precise treatment.

The following results can now be proved in a way analogous to the proofs of earlier theorems as we will indicate.

Theorem 3.6 For every negative integer $-t$, every positive integer a, and every $\epsilon>0$, there exists a positive integer $N$ so that if $n>N$, then $D_{n, a}(x)$ has a root $x_{t}$ satisfying $\left|-t-x_{t}\right|<\epsilon$.

Proof: Analogous to the proof of Theorem 2.3. $\diamond$

Proposition 3.7 Let $v \neq 1$ be a complex number satisfying $|v|=1$, and let a be a positive integer. Then

$$
\lim _{n \rightarrow \infty} \frac{D_{n, a}(v)}{D(n, a)}=0,
$$

where $D(n, a)=D_{n, a}(1)$ is the number of a-derangements of length $n$.

Proof: Analogous to the proof of Proposition 3.4. $\diamond$ 
Theorem 3.8 Let $r$ be an integer so that $0 \leq r<q$, let a be a positive integer, and let $m=\lfloor n / a\rfloor$. Then

$$
\begin{aligned}
\lim _{n \rightarrow \infty} \frac{\sum_{j=0}^{m / q} d_{a}(n, r+j q)}{D(n, a)} & =\lim _{n \rightarrow \infty} \frac{d_{a}(n, r)+d_{a}(n, q+r)+\cdots+d_{a}(n, q\lfloor(m-r) / q\rfloor+r)}{D(n, a)} \\
& =\frac{1}{q}
\end{aligned}
$$

In other words, if $k$ is the number of cycles of a randomly selected $a$-derangement of length $n$, then the probability that $k$ is congruent to $r$ modulo modulo $q$ converges to $1 / q$. Proof: Analogous to the proof of Theorem 3.3. $\diamond$

\section{Further Directions}

As we have mentioned, in the special case of $q=2$, there are known combinatorial proofs for the balanced property of permutations [1], and derangements [7]. It would be interesting to find simple combinatorial proofs for the general case by partitioning almost all permutations (or derangements, or $a$-derangements) of length $n$ into $q$ blocks, and showing that all blocks have the same size, and that almost all permutations of length $n$ got assigned to a block.

The number of cycles is not the only permutation statistic that is interesting when restricted to derangements. See Sections 8 and 9 of [10], and also, [8], [9] for some results on the number of descents, and [4] for some results on the number of excedances.

Finally, $a$-derangements are a special case of a class of permutations, namely the set of all derangements of length $n$ is a union of conjugacy classes in the symmetric group $S_{n}$. It would be interesting to find other such classes for which the balanced property holds. We point out that in [11], another, somewhat similar property is shown for a similar class of permutations.

\section{Acknowledgments}

The author is thankful to Richard Stanley and Herb Wilf for valuable suggestions. He is also indebted to the anonymous referee whose careful reading improved the presentation of the paper, and helped with putting it into context.

\section{References}

[1] M. Bóna, A Walk Through Combinatorics, World Scientific, second edition 2006.

[2] M. Bóna, Combinatorics of Permutations, CRC Press, 2004.

[3] M. Bóna, Introduction to Enumerative Combinatorics, MacGraw-Hill, 2007. 
[4] F. Brenti, Permutation enumeration, symmetric functions, and unimodality. Pacific J. Mathematics, 157 (1993), no. 1, 1-28.

[5] F. Brenti, Combinatorics and Total positivity, J. Combin. Theory Ser. A 71 (1995), no. $2,175-218$.

[6] E. R. Canfield, Asymptotic Normality in Binomial Type Enumeration, Doctoral Dissertation, University of California San Diego, 1975.

[7] R. Chapman, An involution on derangements. 17th British Combinatorial Conference (Canterbury, 1999). Discrete Math. 231 (2001), no. 1-3, 121-122.

[8] J. Désarmenien, M. Wachs, Descentes des dérangements et mots circulaires. Sém. Lotharing. Combin., 19, 13-21, 1984.

[9] J. Désarmenien, M. Wachs, Descent classes on permutations. J. Combin. Theory A, 64 (1993), no. 2, 311-328.

[10] I. Gessel, C. Reutenauer, Counting permutations with given cycle structure and descent set. J. Combin. Theory Ser. A, 64 (1993), 189-215.

[11] B. D. McKay, J. Morse, H. Wilf, The distributions of the entries of Young tableaux, J. Combin. Theory Ser. A 97 (2002), no. 1, 117-128. 


\section{Corrigendum - submitted May 25, 2007}

Lemma 3.5 should be replaced by the Lemma below. The rest of the paper needs no change.

Lemma 3.5 Let $A_{n, a}=d_{a}(n,\lfloor n / 2\rfloor)$ be the leading coefficient of the polynomial $D_{n, a}(x)$. Let $t$ be a fixed positive integer. Then

$$
\lim _{n \rightarrow \infty} \frac{D_{n}(-t)}{A_{n, a}}=0 .
$$

Proof: We first consider $D_{n}(-t)$, then $A_{n, a}$. We then use the same technique to show that the former is small and the latter is big.

Define a function $f$ on the set of positive integers as follows. Let

$$
f(n)=\left\{\begin{array}{l}
0 \text { if } n \leq a, \\
(-t) \text { if } n>a .
\end{array}\right.
$$

Let

$$
h(n)=\sum_{\pi} f\left(\left|\pi_{1}\right|\right) \cdot f\left(\left|\pi_{2}\right|\right) \cdots \cdots \cdot f\left(\left|\pi_{k}\right|\right),
$$

where $\pi$ ranges all permutations of an $n$-element set $[n]$, and $\left\{\pi_{1}, \pi_{2}, \cdots, \pi_{k}\right\}$ is the (unordered) set of cycles of $\pi$. Set $h(0)=1$, and let $E_{h}(x)=\sum_{n \geq 0} h(n) \frac{x^{n}}{n !}$ be the exponential generating function of the sequence $h(n)$.

Then the Exponential Formula, Permutation Version (Corollary 5.1.9 of Enumerative Combinatorics, Volume 2 by Richard Stanley) tells us that

$$
\begin{aligned}
E_{h}(x) & =\exp \left(\sum_{n>a} f(n) \frac{x^{n}}{n}\right) \\
& =\exp \left(-t \sum_{n>a} \frac{x^{n}}{n}\right) \\
& =\exp \left(t\left(\log (1-x)+\sum_{i=1}^{a} \frac{x^{i}}{i}\right)\right) \\
& =(1-x)^{t} \exp \left(t \sum_{i=1}^{a} \frac{x^{i}}{i}\right)
\end{aligned}
$$

Crucially, $D_{n, a}(-t)$ is the coefficient of $x^{n} / n$ ! in $E_{h}(x)$. Indeed, every permutation of length $n$ with $k$ cycles, all of which are longer than $a$, contributes $(-t)^{k}$ to that coefficient. Summing over all values of $k$, our claim follows. Note that $\exp \left(\sum_{i=1}^{a} \frac{x^{i}}{i}\right)$ is just the 
exponential generating function for the number of permutations of length $n$ in which no cycle is longer than $a$. As $t$ is a constant, the factor $(1-x)^{t}$ will not change the growth rate of the coefficient of $x^{n} / n$ ! by more than a polynomial function of $n$.

More precisely, if $\left[x^{n} / n !\right] g(x)$ denotes the coefficient of $x^{n} / n$ ! in the power series $g(x)$, then

$$
\begin{aligned}
\left|D_{n, a}(-t)\right| & =\left|\left[x^{n} / n !\right](1-x)^{t} \exp \left(t \sum_{i=1}^{a} \frac{x^{i}}{i}\right)\right| \\
& \leq t^{n}\left[x^{n} / n !\right]\left|(1-x)^{t} \exp \left(\sum_{i=1}^{a} \frac{x^{i}}{i}\right)\right| \\
& \leq c t^{n} n^{d}\left[x^{n} / n !\right] \exp \left(\sum_{i=1}^{a} \frac{x^{i}}{i}\right),
\end{aligned}
$$

where $c$ is some positive constant, and $d$ is some positive integer.

Let $b$ be a positive integer, and set $m=n / b$. Assume without loss of generality that $m$ is an integer. Then the number of permutations of length $n$ in which each cycle is of length exactly $b$ is

$$
Y(n, b)=\frac{n !}{m ! b^{m}} \sim\left(\frac{n}{e}\right)^{n-m} \sqrt{b} .
$$

In other words, for large $n$, the larger $b$ is, the larger $Y(n, b)$ is, since $m=n / b$.

This, in turn will imply our claim. Indeed, on the one hand, for the leading coefficient $A_{n, a}$ of $D_{n, a}(x)$,

$$
A_{n, a}=Y(n, a+1) \sim\left(\frac{n}{e}\right)^{n-\frac{n}{a+1}} \sqrt{a+1} .
$$

On the other hand, we have seen that $\left|D_{n, a}(-t)\right|$ is not more than a polynomial times the number of permutations of $n$ with no cycles longer than $a$. In order to find an upper bound on the number of such permutations, assign each element of $[n]$ to one cycle length in $n^{a}$ ways, then choose a permutation with equal cycle lengths on each of the $a$ classes of elements created by the assignments. The latter can be done in no more than $Y(n, a)$ ways. Therefore,

$$
\left|D_{n, a}(-t)\right| \leq c n^{d} t^{n} \cdot n^{a} Y(n, a) \sim c t^{n} n^{d+a}\left(\frac{n}{e}\right)^{n-\frac{n}{a}} \sqrt{a} .
$$

Comparing (34) and (35), the proof is now immediate, since the exponent of $n$ is a linear factor larger in (34) than in (35). $\diamond$ 